\title{
Bloqueo de nervios esplácnicos para tratamiento del dolor abdominal crónico benigno. Reporte de caso con revisión temática
}

\section{Splanchnic nerve block for the treatment of benign chronic abdominal pain. a case report and literature review}

\author{
I. H. Salazar Carrera1', R. Romero Andrés², J. P. Revelo Vásconez ${ }^{3}$ y A. E. Mora Useche ${ }^{4}$ \\ ${ }^{1}$ Clínica del Dolor, Sistemas Médicos, Universidad San Francisco de Quito. Cumbayá Pichincha, Ecuador. \\ ${ }^{2}$ Caja Costarricense de Seguro Social. San José, Costa Rica. ${ }^{3}$ Universidad Católica Argentina. Puerto \\ Madero, Buenos Aires, Argentina. ${ }^{4}$ Hospital Carlos Andrade Marín. Quito, Ecuador
}

\section{ABSTRACT}

Chronic abdominal pain of benign origin can be disabling and significantly reduces the quality of life of some patients. Common treatment includes the chronic use of opioids that have also been linked with causing different sources of pain, so alternative pharmacological treatments should be considered instead. However, when these are not effective, insufficient, or cause intolerable adverse effects, interventional pain management should be considered. In this field, the inhibition of splanchnic nerves, which is a technique that can be used to control cancer pain, could play an important role in the treatment of chronic pain of benign origin, despite the lack of solid evidence in its applicability.

As far as we know, it is the first time that this procedure has been performed in Ecuador and this report suggests that it can offer positive results even after the effect of the drugs used has ended.

Key words: Splanchnic nerve block, abdominal pain chronic, visceral pain, splacnic block.

\section{RESUMEN}

El dolor abdominal crónico de origen benigno puede ser incapacitante y disminuye significativamente la calidad de vida de algunos pacientes. Para su tratamiento es usual el uso crónico de opioides que se han asociado a originar otras fuentes de dolor, por lo que deberían sopesar tratamientos farmacológicos alternativos.

Sin embargo, cuando estos no son efectivos, insuficientes u ocasionan efectos adversos intolerables, el manejo intervencionista del dolor debería considerarse. En este campo la inhibición de los nervios esplácnicos que es una técnica que puede ser empleada para el control del dolor oncológico, podría jugar un papel importante a pesar de que aún no cuenta con evidencia sólida de su aplicabilidad en tratar el dolor crónico de origen benigno.

Hasta donde sabemos, en el Ecuador es la primera vez que se realiza este procedimiento y el presente informe sugiere que puede ofrecer buenos resultados, incluso después de terminado el efecto de los fármacos utilizados.

Palabras clave: Bloqueo de nervios esplácnicos, dolor abdominal crónico benigno, dolor visceral, bloqueo esplácnico.
Salazar Carrera IH, Romero Romero A, Revelo Vásconez JP, Mora Useche AE. Bloqueo de nervios esplácnicos para tratamiento del dolor abdominal crónico benigno. reporte de caso con revisión temática. Rev Soc Esp Dolor. 2020;27[4]:273-277
Recibido: 13-05-2020

Aceptado: 30-06-2020

Correspondencia: Iván Hamiyd Salazar Carrera hamiydtrabajo@gmail.com 


\section{INTRODUCCIÓN}

Los nervios esplácnicos torácicos y el plexo celiaco han sido de interés anatómico y clínico por su papel en el manejo del dolor. Los nervios esplácnicos se localizan en la parte media del cuerpo vertebral, lateral a la pleura, ventral al mediastino posterior y caudal a la crura diafragmática.

Los nervios esplácnicos están dentro de un limitado espacio entre la pleura lateralmente, el mediastino posterior ventralmente, las vértebras torácicas lateralmente y las uniones pleurales a la vértebra dorsalmente.

Se originan de las fibras preganglionares simpáticas de T5 a T12, y se definen como mayor (T5-T6 a T9), menor (T10 a T11) y mínimo (T12) (1).

El abordaje transdiscal de nervios esplácnicos fue descrito por Plancarte y cols., en 2003, como una opción más segura y con menos complicaciones a los abordajes tradicionales, sobre todo para alivio del dolor intratable del abdomen superior por causa oncológica [2].

Sin embargo, su aplicación en dolor crónico benigno se ha reportado en la literatura, pero se limita sobre todo al tratamiento del dolor causado por pancreatitis crónica, adherencias viscerales posquirúrgicas, trastornos de motilidad (3) y existen dos reportes de caso exitosos. Uno para el tratamiento del síndrome de encefalopatía neurogastrointestinal mitocondrial (4), y otro para el manejo del síndrome dolor lumbar-hematuria (loin pain-hematuria [LPHS]) [5], siendo por tanto la evidencia aún limitada.

Es así que presentamos el caso del tratamiento exitoso con esta técnica intervencionista avanzada en una paciente con antecedente de dolor crónico severo de 7 años de evolución con excelentes resultados.

\section{MATERIALES Y MÉTODOS}

Se realizó una revisión narrativa no sistemática basada en una búsqueda de literatura en la base de datos PUBMED, que incluyó artículos de metanálisis, revisiones sistemáticas, ensayos clínicos, revisiones narrativas y series de casos. Los términos de búsqueda (MeSH) fueron: "Splanchnic Nerve block", "Abdominal Pain", "Chronic", "Functional” y "Wilkie síndrome".

\section{RESULTADOS}

Se obtuvieron un total de 2.772.167 artículos. La combinación de las 5 palabras clave no arrojó ningún resultado. Se realizó una combinación entre ellas y se obtuvieron los artículos que se tomaron en cuenta. El resto se descartaron por ser estudios en animales, técnica intervencionista diferente, tratamiento intervencionista del dolor en otras regiones anatómicas, estudios en pacientes pediátricos o en dolor oncológico y/o artículos que se repitieron en las diferentes combinaciones (Figura 1).

\section{DESCRIPCIÓN DEL CASO}

Paciente de sexo femenino con una edad de 36 años, con antecedentes patológicos de hipotiroidismo en tra-

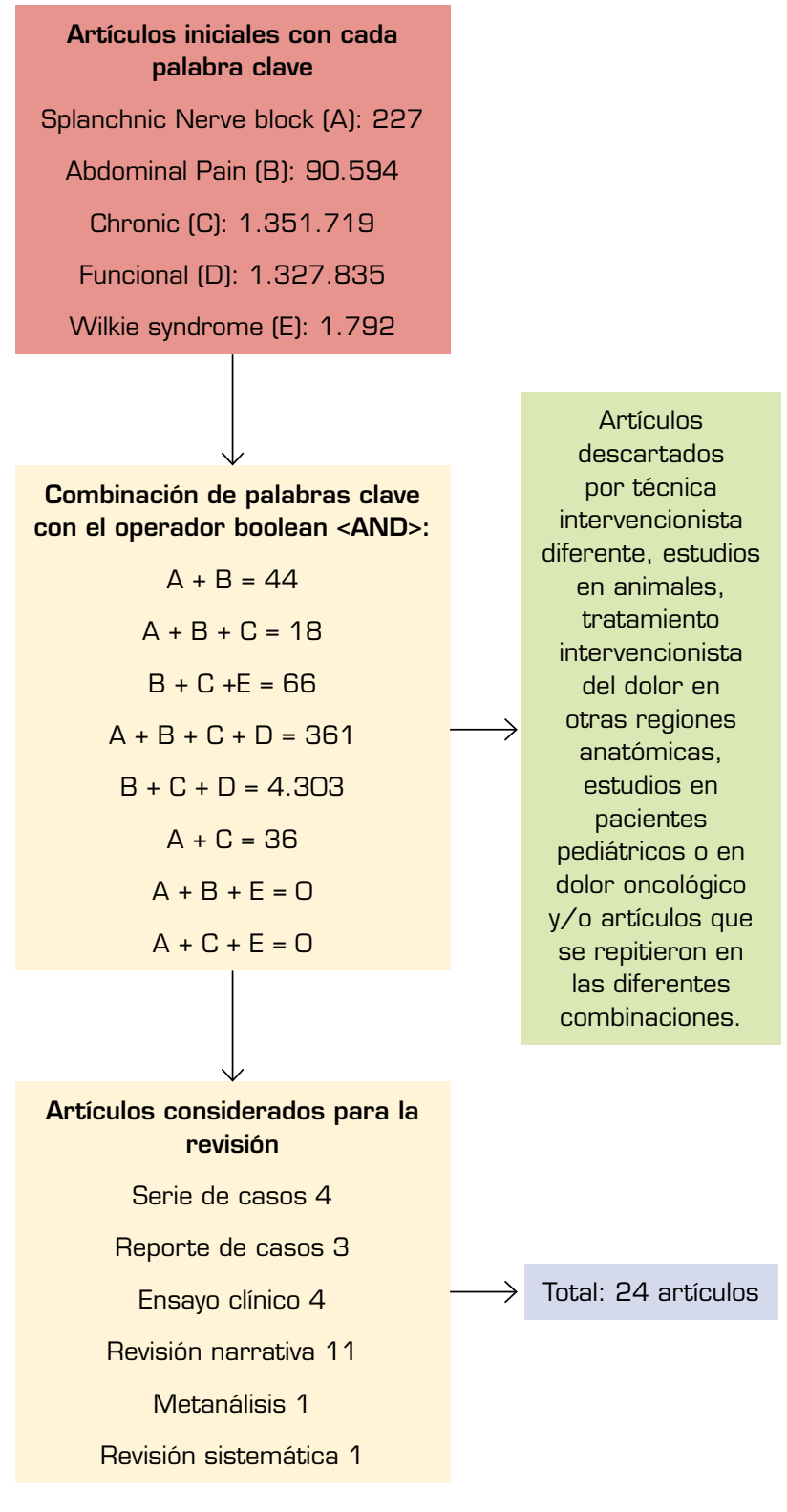

Fig. 1. Flujorama de la búsqueda de artículos.

tamiento con levotiroxina $50 \mu \mathrm{g}$ día, quien inició con cuadro de dolor abdominal hace 6 años. Dolor inicial (2013) tipo pesantez intenso, que llega al vómito y se acompaña de anorexia y pérdida de peso. Se sumó después de un par de meses de evolución de hiperbilirrubinemia por lo que, apoyados en una ecografía que reportó "barro biliar", se decidió realizar colecistectomía laparoscópica. Sin embargo, dolor de iguales características reaparecieron después de 6 horas de terminada la cirugía.

Posteriormente, debido a cuadro de dolor severo, se envió tomografía simple y contrastada de abdomen, con la cual se diagnosticó síndrome de Wilkie, por lo que se sometió al paciente a cirugía abdominal mayor con 
determinación no clara de procedimiento quirúrgico, pero que incluyó sutura y modificación de la anatomía intestinal, además de apendicectomía.

Desde entonces, el cuadro se volvió un círculo vicioso durante dos años de dolor intenso EVA 8-10 e irruptivos 10/10, que requiere múltiples ingresos hospitalarios para analgesia e hidratación, resultados de varios exámenes dentro de la normalidad, múltiples tratamientos farmacológicos y constantes visitas a varios especialistas.

En 2016, debido a cuadro de deterioro progresivo que incluía anemia, bajada de peso en aproximadamente $17 \mathrm{~kg}$ y dolor severo y constante, se decidió realizar laparotomía exploratoria y plastia pilórica. Sin embargo, el dolor intenso abdominal persistió con impacto negativo importante en la calidad de vida, por lo que la paciente visitó a más de 25 especialistas y subespecialistas, incluido psiquiatra, quienes determinan varios diagnósticos y tratamientos, incluido retardo en el vaciamiento gástrico, proponiéndose incluso gastrectomía parcial como posible fuente de dolor, y recibió opioides mayores con mala respuesta.

Nosotros conocemos a la paciente en julio de 2018, quien para entonces tenía tratamiento de buprenorfina parches de 35 microgramos hora cada 72 horas desde hace un año y dos meses, amitriptilina $25 \mathrm{mg}$ v.o. hora sueño, tramadol 15 gotas por razones necesaria (hasta tres veces al día) y tramadol $100 \mathrm{mg}$ i.v. aproximadamente 10 veces en el mes para manejo de crisis de dolor.

La paciente se presentó refiriendo dolor EVA 5-7 constante 10/10 en crisis. Miedo a comer por aumento del dolor y peso de $37 \mathrm{~kg}$, además de importante impacto negativo en la calidad de vida, que fue evaluado con el cuestionario EuroQol 5D- 5L.

Al examen físico llamó la atención la astenia, facies álgidas y dolor moderado a la palpación en parte superior del abdomen.

Es así que replanteamos todo el caso clínico, se realizaron exámenes que incluyeron una serie gastroduodenal, TAC, RM contrastadas de abdomen, los mismos que descartaron dolor de origen oncológico y confirmaron retardo en el vaciamiento gástrico, anastomosis duodeno yeyunal y cambios cicatrizales postquirúrgicos.

Catalogamos el dolor como un síndrome doloroso nociceptivo visceral de causa multifactorial debido a postquirúrgico, funcional y farmacológico (estreñimiento y disfunción de esfínteres].

Es así que se determinó que la solución no es quirúrgica por medio de interconsulta a subespecialista, y que la paciente podría beneficiarse de un bloqueo diagnóstico pronóstico de nervios esplácnicos.

\section{Descripción del tratamiento intervencionista del dolor}

Se realizó un abordaje transdiscal de los nervios esplácnicos a nivel de T10-T11 guiado por tomografía.

Con la paciente en decúbito prono se identificó el espacio seleccionado, se infiltró lidocaína al $1 \%$ a $5 \mathrm{~cm}$ de la línea media izquierda y se avanzó una aguja espinal número 22 progresivamente, realizando cortes topográficos hasta alcanzar el retromediastino. Se confirmó la punta de la aguja con $2 \mathrm{ml}$ de medio de contras- te iopromida $300 \mathrm{mg} / \mathrm{ml}$ [Ultravist ${ }^{\circledR}$ ), y se procedió a inyectar bupivacaína 0,5\% más metilprednisolona $80 \mathrm{mg}$ para un total de $10 \mathrm{ml}$ (Figura 2).

Se dio por terminado procedimiento sin accidentes ni incidentes.
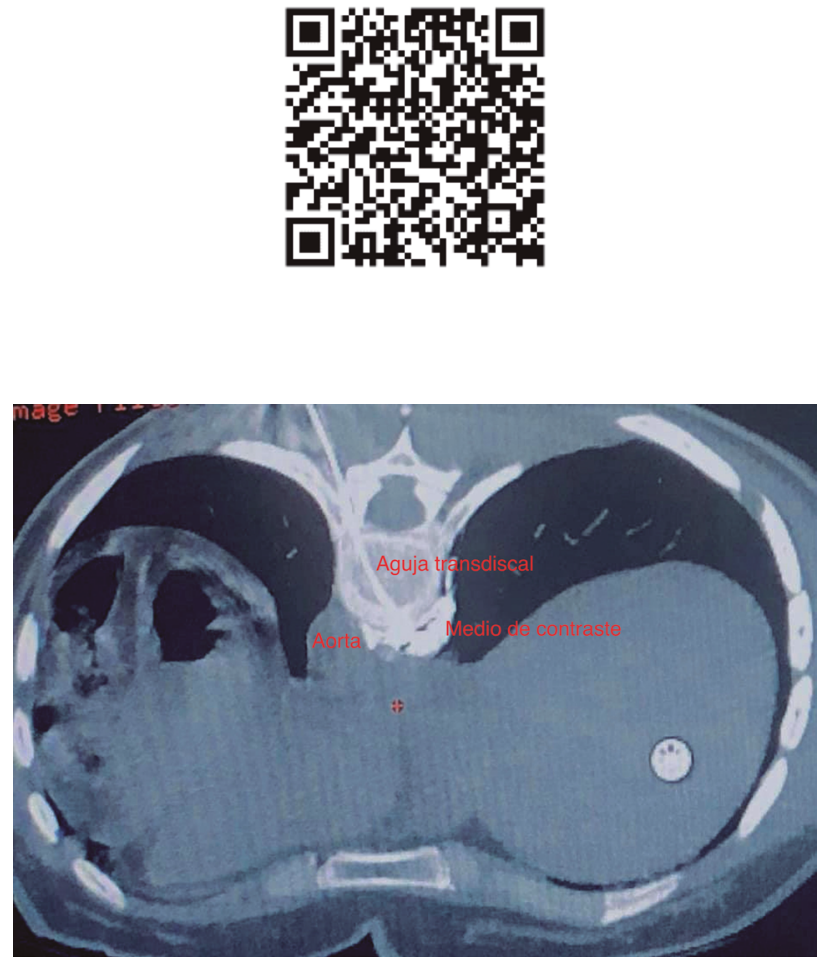

Fig. 2. Aguja a través del disco intervertebral en el espacio T10-T11 guiado por tomografía. Se observa el medio de contraste y medicamentos desplazando la aorta.

\section{Evolución del cuadro clínico}

En la primera consulta posprocedimiento a los 7 días, la paciente se presentó con EVA 2-3/10, sin haber presentado crisis de dolor y con mejoría de las condiciones generales, por lo que desde entonces, y en el transcurso de tres meses, se fue reduciendo progresivamente toda la medicación analgésica.

En el seguimiento a 1 año, la paciente sigue sin presentar crisis de dolor, refiere EVA 0/10 y ha aumentado de peso en $13 \mathrm{~kg}$. Se realizó un nuevo tránsito intestinal que refiere vaciamiento gástrico normal, y ha retomado su vida normal.

\section{DISCUSIÓN CON REVISIÓN TEMÁTICA}

El dolor abdominal visceral crónico de origen benigno puede ser de causa multifactorial incluido el postoperatorio, el dolor funcional, dolor por disfunción del esfínter de Oddi, asociado a opioides, a algunos síndromes 
como el de Wilkie y acompañado a sensibilización central y cambios plásticos, como creemos fue el caso de nuestra paciente [6].

Respecto al manejo farmacológico con opioides para el dolor abdominal crónico benigno faltan pruebas de eficacia, ya que la evidencia respalda su efectividad solo para su uso a corto plazo $[<3$ meses]. Además, un número creciente de estudios informa de dolor abdominal persistente en pacientes con uso crónico de opioides, además de la posible disfunción intestinal inducida por opioides que se manifiesta con dolor abdominal, estreñimiento, náuseas, gases, íleo paralítico, contracción de la vesícula biliar y reflujo gastroesofágico. Y también pueden producir síndrome de intestino narcótico, definido como dolor abdominal crónico o recurrente frecuentemente tratado con dosis altas de opioides [7-9].

Asimismo, existe una relación demostrada entre el uso de opioides con la disfunción de los esfínteres. Se ha concluido, por ejemplo, que la disfunción del esfínter de Oddi y dolor puede suceder luego de la colecistectomía o el uso de tramadol (10-12).

Dichas entidades se encontraban presentes, en mayor o menor grado, en el cuadro clínico previo de nuestra paciente, y podrían explicar la gran mejoría del dolor posterior al retiro de los opioides.

Es así que debemos considerar que hay evidencia cada vez más creciente de que el dolor abdominal crónico benigno podría beneficiarse de terapias farmacológicas alternativas a los opioides como los antiespasmódicos, antagonistas de los receptores 5-HT3, antidepresivos tricíclicos, antipsicóticos como la quetiapina, y anticonvulsivantes como gabapentina y la pregabalina, que pueden ser mejores alternativas al uso de opioides [13-15].

Además, se debería tener siempre presente el manejo intervencionista del dolor cuando los fármacos no brindan analgesia efectiva o producen efectos adversos importantes, ya que constituye un escalón más de la escalera analgésica de la OMS, y se convierte en un importante recurso (16), como lo fue en este caso, donde se realizó un bloqueo de los nervios esplácnicos guiado por tomografía que ofreció gran resolución espacial y ubicación exacta de la punta de la aguja en la reconstrucción 3D. La vía transdiscal fue elegida por ser la técnica que mejor manejamos al ser alumnos directos de quien la ideó (2); teóricamente, por su acceso puede disminuir el riesgo de complicaciones como paraplejia, neumotórax y perforación hepática o renal, sobre todo en pacientes con anatomía modificada. Asimismo, al ser un bloqueo retrocrural nos ofrece una importante barrera anatómica vs. el bloqueo del plexo celiaco [17].

El abordaje de los nervios esplácnicos ha sido de interés anatómico y clínico para el control del dolor, sobre todo en cánceres abdominales, incluido el de páncreas y estómago, además de la pancreatitis (18). Sin embargo, pueden beneficiarse también pacientes que sufren dolor abdominal crónico no maligno (19). Aunque la evidencia en este campo aún sigue siendo escasa, a pesar de que un número no determinado de pacientes sufren este modelo de dolor, que incluso es incapacitante y refractario a tratamientos convencionales.

Existe un reporte retrospectivo reciente de serie de casos con 16 pacientes que resultó interesante, ya que concluyó que el bloqueo de nervios esplácnicos es incluso más efectivo que el bloqueo del plexo celiaco para dolor abdominal crónico benigno, lo que nos hace pensar que incluso se trataría del procedimiento intervencionista de elección en pacientes con esta dolencia (3). No obstante, son necesarios más estudios comparativos para confirmar esta teoría.

Otro punto importante que hay que tener muy en cuenta es la necesidad de "romper el ciclo del dolor". Es decir, que en el tratamiento del dolor crónico permita "descansar" a las neuronas de la constante descarga que perpetúa el dolor, que promueve que se produzca sensibilización central, además de cambios plásticos en el sistema nervioso central, así como la reorganización funcional en la corteza insular y demostradas alteraciones en el perfil bioquímico del cerebro [20-22]. Los mismos que después de un periodo de inactividad podrían mejorar con la consecuente mejoría en el dolor a largo plazo, como se ha evidenciado en nuestra paciente.

En el caso presentado hemos tenido una respuesta excelente a un bloqueo diagnóstico pronóstico, que consideramos es el primer paso en el manejo intervencionista del dolor por tratarse de un dolor no oncológico.

Con los antecedentes antes expuestos, esperamos no recurrir a un procedimiento intervencionista neurolitico. Sin embargo, si fuera necesario, se podría ofrecer una ablación con radiofrecuencia de nervios esplácnicos traspolando la evidencia de serie de casos para dolor por pancreatitis crónica y dolor abdominal funcional con resultados alentadores $[23,24]$ vs. neurosis química con fenol, en la cual tenemos amplia experiencia en modelos de dolor oncológico del abdomen superior.

\section{CONCLUSIONES}

El dolor abdominal crónico de origen benigno puede ser un modelo de dolor incapacitante y que disminuye significante la calidad de vida de algunos pacientes.

Para su tratamiento es usual el uso crónico de opioides, sin embargo el mismo está asociado a otros problemas que incluso pueden ser causa de dolor, como son el síndrome de intestino narcótico abdominal y la disfunción de esfínteres. Por lo que se deben considerar tratamientos alternativos basados por ejemplo en antagonistas de los receptores 5-HT3, antidepresivos tricíclicos, antipsicóticos y/o anticonvulsivantes.

Cuando el tratamiento farmacológico no es efectivo o insuficiente, debe considerarse un manejo intervencionista del dolor, especialmente el bloqueo de nervios esplácnicos por vía transdiscal el mismo que puede ofrecer buenos resultados incluso después de terminado el efecto de los fármacos usados en un bloqueo diagnóstico pronóstico. Además, si este bloqueo es efectivo (reducción de por lo menos el $50 \%$ del dolor) pero corto en el tiempo, se podría considerar una neurólisis química o por radiofrecuencia.

\section{RECOMENDACIONES}

En caso de dolor del abdomen superior de origen crónico benigno y refractario se debe considerar el manejo intervencionista del dolor mediante bloqueo de los nervios esplácnicos por vía transdiscal. 


\section{CONFLICTO DE INTERESES}

Los autores declaran que no existe ningún conflicto de intereses.

\section{FINANCIACIÓN}

La financiación de este trabajo se realizó con recursos propios de los investigadores

\section{AGRADECIMIENTOS}

Expresamos nuestro especial agradecimiento a Sistemas Médicos de la Universidad San Francisco de Quito (SIME - USFQ) por todo el apoyo y ser cede de la Clínica del Dolor en donde se abordó este caso.

\section{BIBLIOGRAFÍA}

1. Loukas M, Klaassen Z, Merbs W, Tubbs RS, Gielecki J, Zurada A. A review of the thoracic splanchnic nerves and celiac ganglia. Clin Anat. 2010;23(5):512-22. DOI: 10.1002/ ca.20964.

2. Plancarte-Sánchez R, Máyer-Rivera F, Guillén Núñez MR, Guajardo-Rosas J, Acosta-Quiroz CO. Abordaje transdiscal de los nervios esplácnicos Cir Ciruj. 2003;71(3):192-203.

3. Kapural L, Lee N, Badhey H, McRoberts WP, Jolly S. Splanchnic block at T11 provides a longer relief than celiac plexus block from nonmalignant, chronic abdominal pain. Pain Manag. 2019;9(2):115-21.

4. Celebi N, Sahin A, Canbay O, Uzümcügil F, Aypar U. Abdominal pain related to mitochondrial neurogastrointestinal encephalomyopathy syndrome may benefit from splanchnic nerve blockade. Paediatr Anaesth. 2006;16(10):1073-6. doi: 10.1111/j.1460-9592.2006.01918.x

5. Moeschler SM, Hoelzer BC, Eldrige JS. A patient with loin hematuria syndrome and chronic flank pain treated with pulsed radiofrequency of the splanchnic nerves. Clin J Pain. 2013;29(11):e26-e29. DOI: 10.1097/ AJP.Ob013e31828c8922.

6. Fernández López MT, López Otero MJ, Bardasco Alonso ML, Álvarez Vázquez P, Rivero Luis MT, López Barros G. Sindrome de Wilkie: a propósito de un caso. Nutr Hosp. 2011;26(3):646-9. DOI: 10.3305/nh.2011.26.3.5215.

7. Szigethy E, Knisely M, Drossman D. Opioid misuse in gastroenterology and non-opioid management of abdominal pain. Nat Rev Gastroenterol Hepatol. 2018;15(3):168-80. DOI: 10.1038/nrgastro.2017.141.

8. Cook SF, Lanza L, Zhou X, Sweeney CT, Goss D, Hollis K, et al. Gastrointestinal side effects in chronic opioid users: results from a population-based survey. Aliment Pharmacol Ther. 2008;27(12):1224-32. DOl: 10.1111/j.13652036.2008.03689.x

9. Tuteja AK, Biskupiak J, Stoddard GJ, Lipman AG. Opioidinduced bowel disorders and narcotic bowel syndrome in patients with chronic non-cancer pain. Neurogastroenterol Motil. 2010;22(4):424-e96. DOI: 10.1111/j.13652982.2009.01458.x.
10. Bistritz L, Bain VG. Sphincter of Oddi dysfunction: Managing the patient with chronic biliary pain. World J Gastroenterol. 2006;12(24):3793-802. DOI: 10.3748/wjg.v12. i24.3793.

11. Wu SD, Zhang ZH, Jin JZ, Kong J, Wang W, Zhang Q, et al. Effects of narcotic analgesic drugs on human Oddi's sphincter motility. World J Gastroenterol. 2004;10(19):2901-4. DOI: 10.3748/wjg.v10.i19.2901.

12. Afghani E, Lo SK, Covington PS, Cash BD, Pandol SJ. Sphincter of Oddi Function and Risk Factors for Dysfunction. Front Nutr. 2017;4:1. D0I: 10.3389/fnut.2017.00001.

13. Gurusamy KS, Lusuku C, Davidson BR. Pregabalin for decreasing pancreatic pain in chronic pancreatitis. Cochrane Database Syst. Rev 2016;2:CD011522. DOI: 10.1002/14651858.CD011522.pub2.

14. Xie $C$, Tang $Y$, Wang $Y, Y u T$, Wang $Y$, Jiang $L$ et al. Efficacy and safety of antidepressants for the treatment of Irritable Bowel Syndrome: a meta-analysis. PLoS ONE. 2015;10(8); e0127815. DOI: 10.1371/journal.pone. 0127815.

15. Daghaghzadeh $H$, Naji F, Afshar $H$, Sharbafchi MR, Feizi A, Maroufi $\mathrm{M}$, et al. Efficacy of duloxetine add on in treatment of inflammatory bowel disease patients: a double-blind controlled study. J Res Med Sci. 2015;20(6):595-601. DOI: 10.4103/1735-1995.165969.

16. Plancarte Sánchez R, Guajardo Rosas J, Guillen Nuñez R. Manejo Integral del Dolor. Cancerología. 2006;1:273-81.

17. Herrero Trujillano M, Mendiola de la Osa A, Insausti Valdivia J, Pérez-Cajaraville J. Revisión de los procedimientos intervencionistas neurolíticos en el dolor asociado al cáncer de páncreas. Propuesta de algoritmo. Rev Soc Esp Dolor. 2019;26(6):34258. DOI: 1020986/resed20193715/2018.

18. Kapural L, Jolly S. Interventional Pain Management Approaches for Control of Chronic Pancreatic Pain. Curr Treat Options Gastroenterol. 2016;14(3):360-70. DOI: 10.1007/ s11938-016-0100-4.

19. Radiofrequency thermocoagulation of the thoracic splanchnic nerve in functional abdominal pain syndrome -A case report. Korean J Anesthesiol. 2011;61(1):79-82. DOI: 10.4097/ kjae.2011.61.1.79.

20. Olesen SS, Frøkjær JB, Lelic D, Valeriani M, Drewes AM. Pain-associated adaptive cortical reorganisation in chronic pancreatitis. Pancreatology. 2010;10(6):742-51. DOI: 10.1159/000321644

21. Woolf CJ. Central sensitization: implications for the diagnosis and treatment of pain. Pain. 2011;152(3 Suppl):S2-S15. DOl: 10.1016/j.pain.2010.09.030.

22. Zhao X, Xu M, Jorgenson K, Kong J. Neurochemical changes in patients with chronic low back pain detected by proton magnetic resonance spectroscopy: A systematic review. Neuroimage Clin. 2016;13:33-8. DOI: 10.1016/j. nicl.2016.11.006.

23. Garcea G, Thomasset S, Berry DP, Tordoff S. Percutaneous splanchnic nerve radiofrequency ablation for chronic abdominal pain. ANZ J Surg. 2005;75(8):640-4. DOI: 10.1111/j.1445-2197.2005.03486.x.

24. Choi JW, Joo EY, Lee SH, Lee CJ, Kim TH, Sim WS. Radiofrequency thermocoagulation of the thoracic splanchnic nerve in functional abdominal pain syndrome -A case report-. Korean J Anesthesiol. 2011;61(1):79-82. DOI: 10.4097/ kjae.2011.61.1.79. 